Case Report

\title{
Pleomorphic leiomyosarcoma of colonic mesentery: an unusual cause of lower GI bleed - case report and literature review
}

\author{
Vandana Singh*, Lalit Aggarwal, Priya Hazrah, Shadan Ali, Ashok Kumar, Anup Mohta
}

Department of General Surgery, Lady Hardinge Medical College, New Delhi, India

Received: 02 November 2020

Revised: 15 January 2021

Accepted: 18 January 2021

\author{
*Correspondence: \\ Dr. Vandana Singh, \\ E-mail: vandana.chauhan51@gmail.com
}

Copyright: () the author(s), publisher and licensee Medip Academy. This is an open-access article distributed under the terms of the Creative Commons Attribution Non-Commercial License, which permits unrestricted non-commercial use, distribution, and reproduction in any medium, provided the original work is properly cited.

\begin{abstract}
Mesenteric masses are infrequent lesions ranging from benign cyst to aggressive malignancies and often present as diagnostic and therapeutic challenge. The mesentery is a frequent recipient of metastasis from the gastrointestinal tract, pancreas, and biliary cancers. Primary mesenteric tumours are relatively rare, mostly mesenchymal in origin and benign in nature. Examples include gastrointestinal stromal tumours and smooth muscle tumours. Pleomorphic leiomyosarcoma of mesocolon is extremely rare with a reported incidence of 1:350,000. So accurate preoperative diagnosis of mesenteric soft tissue tumours is generally difficult. It accounts for less than $1 \%$ of the malignant tumours found in colon. Leiomyosarcoma is a malignant tumour arising from smooth cell lineage. These tumours occur most commonly in middle aged individuals. We describe a case of pleomorphic leiomyosarcoma arising from the colonic mesentery in a 27-year-old male patient, with massive lower gastrointestinal bleed (LGI bleed) causing drop in haemoglobin level from $9 \mathrm{mg} / \mathrm{dl}$ to $6 \mathrm{mg} / \mathrm{dl}$. Ultrasonography and CECT abdomen suggestive of $(17.5 \times 11.6 \times 10.6) \mathrm{cm}$ mass in left side upper abdomen in splenic hilar region. Left hemicolectomy with excision of mass with splenectomy and distal pancreatectomy done. The diagnosis was based on histopathological evaluation using immunohistochemistry (IHC). Histopathological report suggestive of pleomorphic leiomyosarcoma with SMA and vimentin positivity on immunohistochemistry but CD 34 and CD 117 were negative, differentiating it from GIST.
\end{abstract}

Keywords: Leiomyosarcoma, Mesentery, Lower gastrointestinal bleed

\section{INTRODUCTION}

Leiomyosarcoma is a malignant mesenchymal tumour originating from smooth muscle cells typically of uterine, gastrointestinal or soft tissue origin. Leiomyosarcomas arising from each site has unique clinical, radiological and histological features. ${ }^{1}$ The underlying mechanism of development remain unclear. Leiomyosarcomas arise from smooth muscle of blood vessels in the mesentery. Majority of them arise from stomach and small intestine mesentery. However primary pleomorphic leiomyosarcoma of mesocolon with LGI bleeding are extremely rare. These tumours are highly aggressive and surgery is probably best option for treatment. Because of rarity of tumour, standard chemotherapy regimen is not yet established.

\section{CASE REPORT}

A 27-year-old gentlemen presented with complaints of pain in left upper abdomen for two months with a progressively increasing abdominal mass since one month, associated with complaints of anorexia, weight loss and chronic constipation. Single episode of massive rectal bleeding causing drop in haemoglobin level from 9 
$\mathrm{mg} / \mathrm{dl}$ to $6 \mathrm{mg} / \mathrm{dl}$ necessitated multiple blood transfusions for resuscitation.

USG abdomen showed an irregular, solid- cystic mass with internal vascularity (Figure 1). CECT abdomen showed $17.5 \times 11.6 \times 10.6 \mathrm{~cm}$ mass in left upper abdomen at splenic hilar region, superiorly indenting stomach, medially invading pancreatic tail, laterally compressing spleen and inferiorly upper pole of left kidney and was inseparable from descending colon. No metastasis to liver.
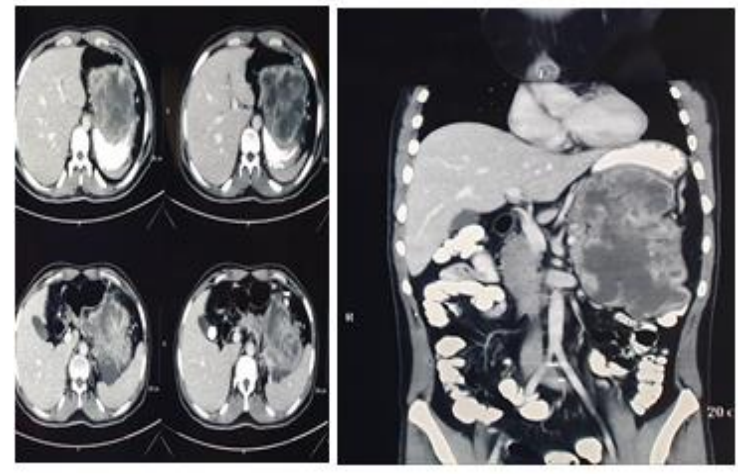

Figure 1: CECT abdomen showing mass with its relation to surrounding structures.

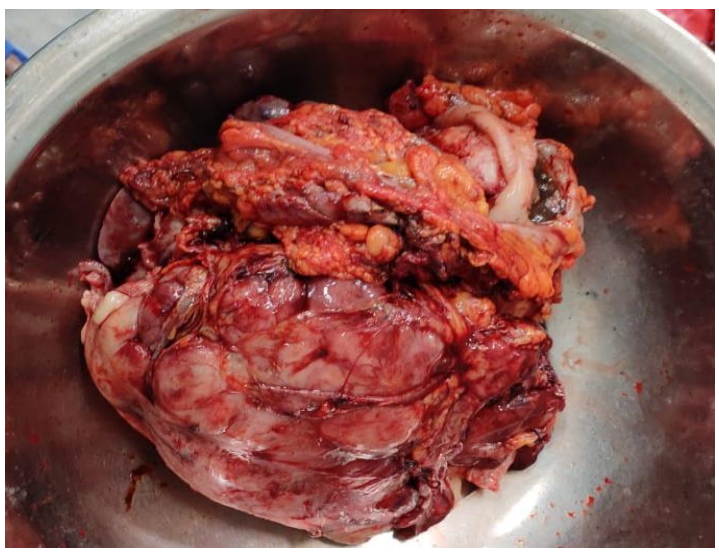

Figure 2: Gross appearance of the tumour.

On exploratory laparotomy there was a large mass $(20 \times 18 \times 9) \mathrm{cm}$, at splenic flexure of colon, encasing splenic hilum and distal pancreas, abutting posterior surface of stomach and upper pole of left kidney (Figure 2 ). Left hemicolectomy with excision of mass with splenectomy and distal pancreatectomy was done and specimen was sent for histopathological examination. On gross examination the tumour measured $18 \times 18 \times 7 \mathrm{~cm}$. Cut section of lesion showed solid mass with necrotic areas (Figure 3). Histopathological report was suggestive of pleomorphic leiomyosarcoma of colonic mesentery with SMA and vimentin positive on IHC and negative for CD 117, CD 34. Spleen and pancreas were uninvolved by tumour.
Patient was discharged 5 days later and kept on follow up. Patient received two cycles of gemcitabine chemotherapy. Patient developed symptoms of intestinal obstruction after five months of surgery with CECT abdomen findings suggestive of liver metastasis and died six months after surgery due to progressive disease.

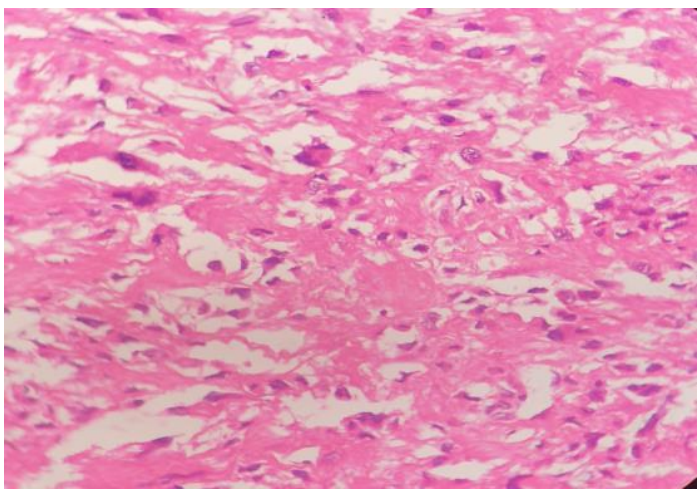

Figure 3: Storiform appearance with mitosis.

\section{DISCUSSION}

The mesentery presents a common site for the metastasis of gastrointestinal malignancies via lymphatics. However, the primary tumour arising in mesentery is extremely rare, lymphomas being the most common followed by mesenchymal tumors. ${ }^{2}$ These malignancies include GIST, leiomyosarcomas, liposarcoma, fibrosarcoma, pleomorphic undifferentiated sarcomas and malignant teratomas. ${ }^{3}$ Of these tumours leiomyosarcomas are most common type with a reported incidence of 1:350000 and were first described by Yannopoulos and Stout. ${ }^{3,4}$

These tumours primarily affect middle aged persons. Common presenting complaints includes a palpable abdominal mass and pain. But infrequently abscess, acute intraperitoneal haemorrhage, or obstructive ileus may also be the presenting features. Most of these tumours tend to be asymptomatic and grow to a large size by the time of diagnosis since the mobility and elasticity of mesentery allow the tumour to occupy a large intraperitoneal space without causing obstructive symptoms. Preoperative imaging is of paramount importance in the management of such patients. Both CECT abdomen and ultrasound contribute toward detection, characterization, and potential surgical planning for mesenteric lesions. On CECT, most of the solid masses are neoplastic in nature and cystic ones are benign. Cystic masses usually have a thin wall and lack solid components, which can help discriminate them from GISTs and sarcomas. In the comparison of GISTs and sarcomas, the former tend to be less invasive in appearance. $^{5}$

Percutaneous biopsy is contraindicated because skin metastasis and peritoneal dissemination may be induced by biopsy. A definitive diagnosis can only be made by 
histopathological study (IHC staining) and genetic analysis. Treatment strategies for mesenteric leiomyosarcoma vary. Surgical excision with a wide margin of normal tissue is most effective since adjuvant chemotherapy or radiotherapy are unreliable. In our case en bloc resection was done and the margins were free from tumour. These patients generally display a poor prognosis. The overall 5-year survival rate for these tumour is only $20-30 \%$, and complete primary surgical resection is critical for achieving the best outcome. Recurrence can occur within 5 years, so long term follow-up of such patients for 5 years or more, with particular attention to gastrointestinal tract, liver, and lung, is necessary. Survival is influenced by histological grade of mesenteric leiomyosarcomas, based on cell differentiation, cellularity of tumour, anaplasia, and number of mitoses per HPF. It is differentiated from GIST on basis of IHC markers only. Due to its rarity and low incidence, the standard chemotherapy regimen is not yet established.

\section{CONCLUSION}

Mesenteric masses often pose a diagnostic and therapeutic challenge. Diagnosis can only be confirmed by histopathology and immunological staining. Even though surgery is the main modality of treatment recurrence is common in these tumours. Early detection and wide surgical resection will improve prognosis of these lesions.
Funding: No funding sources Conflict of interest: None declared Ethical approval: Not required

\section{REFERENCES}

1. Weiss SW, Goldblum JR. Leiomyosarcoma. In: Weiss SW, Goldblum JR, editors. Enzinger and Weiss's Soft Tissue Tumors. 5th edition. Philadelphia: Mosby; 2008:769-88.

2. Sharma R, Mahajan N, Vij A, Chaudhary UK, Sharma A. Mesenteric Leiomyosarcoma Mistaken as Subserosal Fibroid: A Rare Case Report. Int J Syst Sci. 2015;2:8-10.

3. Yannopoulos K, Stout AP. Primary Solid Tumors of the Mesentery. Cancer. 1963;16:914-27.

4. Sidhic AK, Ranjith M, Ali KP, Tej PR. Leiomyosarcoma of the mesentry, a rare mesentric tumour. Int J Surg Case Rep. 2015;7:58-60.

5. Katz SC, DeMatteo RP. Gastrointestinal stromal tumors and leiomyosarcomas. J Surg Oncol. 2008;97:350-9.

Cite this article as: Singh V, Aggarwal L, Hazrah P, Ali S, Kumar A, Mohta A. Pleomorphic leiomyosarcoma of colonic mesentery: an unusual cause of lower GI bleed - case report and literature review. Int Surg J 2021;8:754-6. 